\title{
Gefordert ist mehr Effizienz im Gesundheitswesen
}

\author{
Judith Trageser, Anna Vettori, Rolf Iten, Juliane Fliedner
}

INFRAS

\begin{abstract}
Durch ein Set von mehreren im Ausland praktizierten Lösungsansätzen könnte die Effizienz des schweizerischen Gesundheitswesens gesteigert werden. Es braucht stärkere Anreize auf Seiten der Leistungserbringer sowie mehr Evidenz über die Wirksamkeit. Zu diesem Schluss kommt eine INFRAS-Studie, die ausgewählte Lösungsansätze beleuchtet. Sie zeigt Effizienzpotentiale auf und geht der Frage nach, wie sie in der Schweiz erfolgreich umgesetzt werden könnten.
\end{abstract}

\section{Ineffizienzen im schweizerischen Gesundheitswesen}

«Gut, aber teuer» wird heute vielfach über das schweizerische Gesundheitswesen geurteilt. In der Tat zeigen verschiedene Studien, dass trotz der ausgewiesenen hohen Qualität durchaus Effizienzpotentiale im schweizerischen Gesundheitswesen bestehen. Ineffizienzen, die aus der mangelnden Koordination der Versorgung, der Überversorgung mit nicht notwendigen Leistungen oder einer überhöhten Nachfrage der Versicherten resultieren, machen rund 10\% der gesamten jährlichen Gesundheitsausgaben aus [1]. Der Bundesrat geht in seinem Bericht "Gesundheit 2020» davon aus, dass 20\% der Kosten durch ein effizienteres Gesundheitssystem eingespart werden könnten.

Vor diesem Hintergrund untersuchte die INFRASStudie* «Mehr Effizienz im Gesundheitswesen - ausgewählte Lösungsansätze» [2] mögliche Stossrichtun-

Anreize Anbieter Anreize Nachfrager

\begin{tabular}{|c|c|c|}
\hline $\begin{array}{l}\text { Ebene } \\
\text { Finanzierung } \\
\text { Vergütung }\end{array}$ & $\begin{array}{l}\text { - Leistungsorientierte Vergütung } \\
\text { - Bundled Payment } \\
\text { - Rechenschaftspflichtige } \\
\text { Versorgungsorgorganisationen } \\
\text { - HTA } \\
\text { - Nutzenorient. Vergütung Medikam. }\end{array}$ & $\begin{array}{l}\text { - Medical Savings Accounts } \\
\text { - Personal Health Budgets }\end{array}$ \\
\hline $\begin{array}{l}\text { Ebene } \\
\text { Organisation }\end{array}$ & $\begin{array}{l}\text { - Optimierung station. Versorg.strukt. } \\
\text { - Integrierte } \\
\text { Versorgungsorganisationen } \\
\text { - Medizinische Guidelines } \\
\text { - Optimierung Skill-Mix } \\
\text { - «E-Health» }\end{array}$ & $\begin{array}{l}\text { - Förderung Selbstmanagement } \\
\text { - Steuerung Patientenströme } \\
\text { - Stärkung Präv. + Ges.förderung }\end{array}$ \\
\hline
\end{tabular}

Abbildung 1: Typologie möglicher Lösungsansätze. gen, mit denen bedeutende Ineffizienzen reduziert werden könnten. Die Studie fokussiert auf Lösungsansätze, die im Ausland gut funktionieren, in der Schweiz aber noch wenig Beachtung finden.

\section{Lösungsansätze - ein Blick ins Ausland}

Im Ausland wird bereits vieles erprobt und umgesetzt, um Ineffizienzen im Gesundheitswesen zu eliminieren. Dabei geht es u.a. darum, Transparenz über die Qualität der Leistungserbringung zu schaffen, die umfassende Versorgung durch integrierte Versorgungsorganisationen zu fördern, Patientinnen und Patienten aktiv einzubeziehen oder ambulante Versorgungsstrukturen zu stärken. Insgesamt hat die vorliegende Studie 15 verschiedene Lösungsansätze zur Effizienzsteigerung identifiziert, die auf unterschiedlichen Systemebenen eingreifen und unterschiedliche Anreize setzen (Abb. 1).

In der Studie wurden fünf der in Abbildung 1 aufgeführten Lösungsansätze vertieft untersucht (schwarz hervorgehobene Lösungsansätze). Basierend auf Literaturanalysen beleuchtet die Studie die Effizienzpotentiale dieser Lösungsansätze. Zudem untersucht die Studie anhand von Interviews mit ausgewählten Stakeholdern, inwiefern die Lösungsansätze in der Schweiz eingeführt bzw. stärker etabliert werden könnten.

\section{Bundled Payment (Komplexpauschalen)}

«Bundled Payment» bezeichnet ein Vergütungsmodell, bei dem alle im Rahmen einer Behandlungsepisode erbrachten Leistungen der verschiedenen 
Leistungserbringer (z.B. ambulanter Arzt, Spital, Reha, Physiotherapie) pauschal abgegolten werden. Durch die gebündelte Vergütung erhalten die Leistungserbringer einen Anreiz, die Anzahl und Kosten der Leistungen, die in der Pauschale enthalten sind, $\mathrm{zu}$ optimieren und diese Leistungen bestmöglich zu koordinieren. Die bisherigen Erfahrungen im Ausland lassen vermuten, dass Bundled Payment die Qualität, Outcomes (Gesundheitszustand) und Kosteneffizienz des schweizerischen Gesundheitswesens prinzipiell verbessern könnte. Grob geschätzt lassen sich mit Bundled Payment bei Knie- und Hüftgelenkersatz, Myokardinfarkt und Diabetes rund 650 Millionen Franken (rund 1\% der Gesundheitsausgaben) einsparen. Das Bundled Payment ist aber auch mit gewissen Gefahren verbunden, wie z.B. dem «Underuse» von angemessenen Leistungen oder einem hohen administrativen Aufwand.

\begin{tabular}{l} 
Was ist Bundled Payment? \\
\hline Pauschale Vergütung ganzer Leistungsbündel, die zu einer \\
Episode gehören. \\
\hline - Ziel: Stärkere Koordination zwischen Leistungserbringern, \\
Reduktion angebotsinduzierter Nachfrage \\
Wo existiert Bundled Payment? \\
\hline - Ausland: USA, NL, SE, UK: chronische Episoden, akutmedi- \\
zinische Episoden \\
- Schweiz: Einzelne Modelle im akutmedizinischen Bereich \\
(v.a. Orthopädie) \\
\hline Was sind Hemmnisse in der CH? \\
\hline - Fehlende Anreize und Vorbehalte, Investitionsaufwand bei \\
Ärztinnen und Ärzten \\
\hline - Fragmentiertes Versorgungssystem \\
\hline - Fehlende Daten und technische Voraussetzungen \\
\hline - Kleine Anzahl Fälle und dadurch hohe Risikostreuung bei \\
Pauschale \\
\hline Welche Faktoren fördern die Anwendung? \\
\hline - Strukturwandel Spitäler (in Richtung mehr Zusammenarbeit \\
mit ambulantem Sektor) \\
\hline - Strategie «eHealth» \\
\hline - Verfeinerung Risikoausgleich (Anreize für Versicherer) \\
\hline
\end{tabular}

\section{Optimierung des Skill-Mix}

Die Idee hinter einer Optimierung des Skill-Mix ist es, Arbeiten und Kompetenzen innerhalb und zwischen den Gesundheitsberufen so umzuverteilen, dass die Leistungen mit minimalem Ressourceneinsatz und mindestens gleichbleibender Qualität erbracht werden. Die empirische Evidenz für den Skill-Mix bei Pflegefachpersonen und Ärzten zeigt, dass sich Optimierung des Skill-Mix vor allem positiv auf die Qualität der Versorgung, die Patientenzufriedenheit und die Gesundheit auswirkt. In Bezug auf mögliche Kosteneinsparungen ist die empirische
Evidenz weniger eindeutig. Dies liegt u.a. daran, dass Skill-Mix in der Praxis zu Leistungsausweitungen führen kann.

\begin{tabular}{l} 
Was ist Skill-Mix? \\
\hline Arbeits- und Kompetenzumverteilung zwischen Gesundheits- \\
berufen (hier: zwischen Grundversorgern und Pflegefach- \\
personen, erweiterte Rollen der Pflege). \\
\hline - Ziel: Geringerer Ressourceneinsatz bei mindestens gleich- \\
bleibender Qualität; Arbeitskräftemangel begrenzen \\
\hline Wo existiert Skill-Mix? \\
\hline - Ausland: Stark verbreitet, insbesondere angelsächsische \\
Länder (Einsatz von Advanced Practice Nurses APN, \\
«nurse-led clinics» etc.) \\
\hline - Schweiz: Einsatz von APN in Spitälern, vereinzelt in Grund- \\
versorgung, einzelne Pilotprojekte \\
\hline Was sind Hemmnisse in der CH? \\
\hline - Vorbehalte Ärztlnnen bzgl. Qualität \\
\hline - Fehlende Abgeltung von Leistungen im Tarifsystem \\
\hline - Fragmentiertes Versorgungssystem \\
\hline - Fehlende gesetzliche Rahmenbedingungen (z.B. Haftpflicht) \\
\hline - Bildungssystem wenig auf Interprofessionalität ausgerichtet \\
\hline Welche Faktoren fördern die Anwendung? \\
\hline - Fachkräftemangel bei Grundversorgern und Pflege \\
\hline - Erfolgreiche Pilotversuche verbessern Akzeptanz \\
\hline Pauschalen (DRGs, Kopfpauschalen) machen Druck auf Kos- \\
\hline -
\end{tabular}

\section{Medizinische Guidelines}

Bei medizinischen Guidelines handelt es sich um evidenzbasierte Empfehlungen, die zum Ziel haben, die Patientenbehandlung zu optimieren und zu standardisieren. Sie sollen helfen, Prozesse effizienter zu gestalten sowie überflüssige Leistungen und überholte Behandlungen zu vermeiden. Sie bekämpfen damit die Überversorgung, eine der grossen Ineffizienzen im schweizerischen Gesundheitswesen. Die Erfahrungen mit Guidelines sind grundsätzlich positiv. Studien zeigen, dass Diagnosen effizienter erstellt werden, es weniger Änderungen bei Medikamenten gibt und Ärztinnen und Ärzte vermehrt prüfen, ob Leistungen notwendig und zweckmässig sind. Die Erkenntnisse zur Kostenwirksamkeit sind hingegen noch schwach, da sie schwierig nachzuweisen sind.

Was sind medizinische Guidelines?

Evidenzbasierte Empfehlungen für Ärztinnen und Ärzte.

- Ziel: Optimierung und Standardisierung der Patientenbehandlung

Wo existieren medizinische Guidelines?

- Ausland: Weit verbreitet in unterschiedlichen Ländern und Fachgebieten, Methodik teilweise institutionalisiert 
- Schweiz: Ansatzweise verbreitet in verschiedenen Fachgebieten, z.B. Kardiologie, Onkologie, Diabetologie, Chirurgie; Erarbeitung durch Fachgesellschaften, spitalinterne Guidelines

\begin{tabular}{l}
\hline Was sind Hemmnisse in der CH? \\
\hline - Mangelnde Kenntnisse, Befürchtung von Ärztlnnen bzgl. \\
eingeschränkter Therapiefreiheit, von ÄrztInnen und Patien- \\
tlnnen bzgl. Unterversorgung \\
\hline - Unzureichende Evidenz über Wirksamkeit \\
- Ungenügende Finanzierung für die Erarbeitung \\
- Fehlende Anreize in der Grundversorgung \\
Welche Faktoren fördern die Anwendung? \\
- Pauschalen (DRGs, Kopfpauschalen) und damit verbunden \\
Druck auf Kosteneffizienz \\
- Strategie «eHealth»
\end{tabular}

\section{Medical Savings Accounts}

Bei Medical Savings Accounts (MSA) handelt es sich um individuelle Gesundheitssparkonten, die der Versicherte äufnet, indem er regelmässige Beiträge einbezahlt. Im Krankheitsfall finanziert er die Ausgaben für die Behandlung aus den selber angesparten Mitteln. Ziel von MSA ist es, die Eigenverantwortung und das Kostenbewusstsein der Versicherten zu stärken. Häufig werden MSA mit einer Versicherung für Hochrisikokrankheiten ergänzt. Studien, die MSA untersuchen, finden allerdings mehrheitlich keine eindeutigen Belege für Kosteneinsparungen. Sie belegen vielmehr, dass MSA sogar negativ wirken können, weil Leistungen zu spät in Anspruch genommen werden. MSA haben allerdings den Vorteil, dass sie die jüngere Generation entlasten, wenn infolge der demographischen Entwicklung die Kosten für die ältere Generation steigen. Auf der anderen Seite sehen verschiedene Experten durch MSA je nach Ausgestaltung das Solidaritätsprinzip gefährdet.

Was sind Medical Savings Accounts?

Individuelle Gesundheitssparkonten, aus denen Krankheitskosten bezahlt werden (ausser Hochrisikofälle).

- Ziel: Stärkung des Kostenbewusstseins von PatientInnen, Verzicht auf unnötige Leistungen, Verbesserung der intergenerationellen Gerechtigkeit

Wo existieren Medical Savings Accounts?

- Ausland: Singapur, China, Südafrika, USA

Was sind Hemmnisse in der $\mathrm{CH}$ ?

- Befürchtungen von Entsolidarisierung

- Keine Evidenz über bessere Qualität oder langfristige Kosteneffizienz

- Fehlende gesetzliche Grundlagen

- Politisch schwer kommunizierbar

Welche Faktoren fördern die Anwendung?

- Demographische Entwicklung und Argument, dass dadurch

jüngere Generation entlastet wird

\section{Förderung des Selbstmanagements}

Interventionen zur Förderung des Selbstmanagements sollen die Patientinnen und Patienten in die Lage versetzen, selbstständig und unabhängig zu leben. Patientenexpertenprogramme beispielsweise bieten spezialisierte Informationen für chronisch Erkrankte und individuelle Patientenschulungen. Der Lösungsansatz zielt darauf ab, einerseits die Gesundheitsversorgung besser an die Bedürfnisse der Patienten anzupassen und andererseits eine unnötige Leistungsinanspruchnahme durch Patienten aufgrund fehlender Informationen zu senken. Verschiedene Beispiele zeigen die positiven Wirkungen auf die Patientenzufriedenheit, die Gesundheitsoutcomes wie z.B. Blutzuckerwerte und eine tiefere Hospitalisationsrate auf. Die Literaturanalyse deutet aber auch darauf hin, dass die Compliance der Patientinnen und Patienten ein Unsicherheitsfaktor für die Wirkungen auf die Outcomes ist.

Was ist Selbstmanagement?

Fähigkeit von PatientInnen, mit der eigenen Erkrankung umzugehen.

- Ziel: Vermeidung von Komplikationen

Wo existieren Massnahmen zur Förderung?

- Ausland: Verschiedene Programme stark verbreitet, z.B. Australien, USA, England

- Schweiz: Verschiedene Programme/Pilotprojekte, z.B. EVIVO-Patientenschulungen, Projekte LEILA-Leben mit Langzeiterkrankung

Was sind Hemmnisse in der $\mathrm{CH}$ ?

- Befürchtungen mangelnder Compliance der PatientInnen

- Fehlende Vergütung der Leistungen, Tarifsysteme

- Fehlende Anreize für Versicherungen

Welche Faktoren fördern die Anwendung?

- Grundsätzlich hohe Akzeptanz

- Steigender Versorgungsbedarf von chronisch Kranken

- Verfeinerung Risikoausgleich

- Strategie «eHealth"

\section{Folgerungen}

- Grössere Effizienzpotentiale durch ein Set an Lösungsansätzen: Der «schlafende Riese», mit dem auf einen Schlag grosse Effizienzpotentiale geweckt werden könnten, scheint aufgrund der Ergebnisse der Studie nicht zu existieren. Dennoch zeigt sich anhand der vertieft untersuchten Lösungsansätze, dass die Effizienz über verschiedene, sich ergänzende Lösungsansätze gesteigert werden kann. Die Potentiale dürften schätzungsweise im einstelligen Prozentbereich liegen.

- Keine grundlegenden Systemumwälzungen notwendig: Viele der untersuchten Lösungsansätze lassen sich gut in das bestehende System einbet- 
ten und in diesem Rahmen umsetzen. Dies betrifft insbesondere die Ansätze, die organisatorische Veränderungen bei den Leistungserbringern (z.B. Optimierung des Skill-Mix, Medizinische Guidelines etc.) voraussetzen. Viele der untersuchten Ansätze sind auch bereits in Ansätzen in der Schweiz vorhanden. Grundlegende Systemumwälzungen sind somit - mit Ausnahme einer Implementierung von MSA - nicht notwendig, um die Effizienz im Gesundheitswesen zu verbessern.

- Patientenbedürfnisse in den Mittelpunkt: Hinter verschiedenen Lösungsansätzen zur Effizienzsteigerung steht die Idee der "value-based care». Diese stellt die Patientenbedürfnisse in den Mittelpunkt. Die Fokussierung auf den Nutzen der Patientinnen und Patienten stellt somit einen wichtigen Treiber dar, nicht nur für die Qualität des Gesundheitswesens, sondern auch zur Steigerung der Kosteneffizienz.

- Realisierung der Effizienzpotentiale abhängig von konkreter Ausgestaltung: Die Analyse zeigt, dass die Lösungsansätze zum Teil auch mit gewissen Gefahren verbunden sind und der «Teufel häufig im Detail steckt». Um die Effizienzpotentiale auch realisieren zu können, kommt es auf die konkrete Ausgestaltung an.

- «Bottom-up»-Entwicklung der Lösungsansätze fördern: Erfahrungen aus dem Ausland zeigen, dass es wenig erfolgversprechend ist, die Lösungsansätze «Top-down» zu verordnen. Wichtig wäre vielmehr, die Leistungserbringer zu motivieren, Lösungsansätze "Bottom-up» zu erarbeiten und durchzusetzen.

Infras

Forschung und Beratung

Binzstrasse 23

CH-8045 Zürich

Tel. 0442059526

judith.trageser[at]infras.ch
Verschiedene Rahmenbedingungen im Gesundheitssystem senden heute noch falsche Signale aus, so zum Beispiel die stark fragmentierte Versorgung in der Schweiz, die in der ambulanten Versorgung herrschende Einzelleistungsvergütung oder die geltenden Tarife, die nicht alle Leistungen abbilden.

- Evidenz und Good Practice notwendig: Trotz positiver Erfahrungen im Ausland bestehen gegen die Lösungsansätze verschiedene Vorbehalte. Diese sind zumindest teilweise darauf zurückzuführen, dass die Stakeholder mangelhaft informiert sind. Damit sich die Lösungsansätze durchsetzen, ist es deshalb wichtig, Evidenz und Good Practice zu fördern.

Es ist wenig erfolgversprechend, die Lösungsansätze «Top-down» zu verordnen.

- Der Bundesrat macht vorwärts: Damit sich neue Lösungsansätze besser etablieren können, hat der Bundesrat bereits einige wichtige Massnahmen in Gang gesetzt. Zu nennen sind hier die Qualitätsstrategie, das Gesundheitsberufegesetz, die Strategie "eHealth", die Verfeinerung des Risikoausgleichs sowie der Ausbau an Versorgungsdaten. Die Strategie Gesundheit2O2O zeigt grundsätzlich, dass der Bundesrat die grössten Herausforderungen und notwendigen Stossrichtungen erkannt hat. Es wird nun darum gehen, diese auch konsequent unter Mithilfe aller Stakeholder umzusetzen.

Referenzen

1 INFRAS, Crivelli 2012. Effizienz, Nutzung und Finanzierung des Gesundheitswesens. Bern: Akademien der Wissenschaften Schweiz; 2012.

2 INFRAS 2014. Mehr Effizienz im Gesundheitswesen. Ausgewählte Lösungsansätze. Abrufbar unter: www.infras.ch/d/projekte/ displayprojectitem.php?id=4929 